\title{
Absorption coefficient dependent non-linear properties of thin film luminescent solar concentrators
}

\author{
S. Chandra \\ Trinity College Dublin, Ireland \\ M. Rafiee \\ Trinity College Dublin, Ireland \\ John Doran \\ Technological University Dublin, john.doran@tudublin.ie
}

See next page for additional authors

Follow this and additional works at: https://arrow.tudublin.ie/scschphyart

Part of the Civil Engineering Commons, Environmental Engineering Commons, and the Physical Sciences and Mathematics Commons

\section{Recommended Citation}

S. Chandra, M. Rafiee, J. Doran, S.J. Mc Cormack, Absorption coefficient dependent non-linear properties of thin film luminescent solar concentrators, Solar Energy Materials and Solar Cells, Volume 182, 2018, Pages 331-338, ISSN 0927-0248, DOI: 10.1016/j.solmat.2018.04.004.

This Article is brought to you for free and open access by the School of Physics \& Clinical \& Optometric Science at ARROW@TU Dublin. It has been accepted for inclusion in Articles by an authorized administrator of ARROW@TU Dublin. For more information, please contact arrow.admin@tudublin.ie, aisling.coyne@tudublin.ie, gerard.connolly@tudublin.ie.

Funder: Higher Education Authority (HEA), Ireland;

European Research Council; Science Foundation Ireland

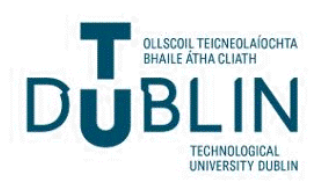




\section{Authors}

S. Chandra, M. Rafiee, John Doran, and S. J. McCormack

This article is available at ARROW@TU Dublin: https://arrow.tudublin.ie/scschphyart/153 


\title{
Absorption coefficient dependent non-linear properties of thin film luminescent solar concentrators
}

\author{
S. Chandra ${ }^{\mathrm{a}, *}$, M. Rafiee ${ }^{\mathrm{a}}$, J. Doran ${ }^{\mathrm{b}}$, S.J. Mc Cormack ${ }^{\mathrm{a}}$ \\ a Dept. of Civil, Structural and Environmental Engineering, Trinity College Dublin, Dublin 2, Ireland \\ ${ }^{\mathrm{b}}$ School of Physics, Dublin Institute of Technology, Kevin Street, Dublin 08, Ireland
}

\section{A R T I C L E I N F O}

\section{Keywords:}

Thin film luminescent solar concentrator (LSC)

Dye

Absorption coefficient

Re-absorption

Resonant energy transfer (RET)

Non-linear properties

Optical efficiency

\begin{abstract}
A B S T R A C T
This research article studied absorption coefficient dependent losses and efficiency of thin film luminescent solar concentrator (LSC). The optimum absorption coefficient was determined by fabricating red dye doped thin film LSCs with varying absorption coefficients of $24-202 \mathrm{~cm}^{-1}$. The optical, electrical, and ray-trace model predicted results were compared to establish the optimum absorption coefficient for fabricated thin film LSCs. The optical and electrical efficiencies of thin film LSCs are a function of absorption coefficient; follow linearly at lower values, gradually becoming non- linear at higher values of absorption coefficient which restricts the overall efficiency. The multi layered ray-trace model predicted results deviated from the experimental results at high absorption coefficient. Non-linearity was introduced by losses mechanism of re-absorption, resonant energy transfer (RET), and scattering in thin film LSCs. Re-absorption losses were found to be dependent on optical pathlength and absorption coefficient of thin film. They were identified and quantified through locally collected emission and optical path-length in thin film LSCs. Re-absorption losses caused a red shift in emission peak by $\approx 20 \mathrm{~nm}$ as the absorption coefficient increased from 24 to $202 \mathrm{~cm}^{-1}$. RET decreased fluorescence life time of dye from $5 \mathrm{~ns}$ to $2 \mathrm{~ns}$ for the absorption coefficient increased from $24-202 \mathrm{~cm}^{-1}$. These absorption coefficient dependent losses limit the optical and electrical efficiency of thin film LSC devices, and compared with bulk LSC.
\end{abstract}

\section{Introduction}

Luminescent solar concentrator (LSCs) technology was proposed in the late 1970s [1-3] as a means to concentrate solar radiation onto a smaller area of solar cell, and hence, enhance the solar cell output. The main objective of this technology is to replace the large area of solar cells in a standard flat-plate photovoltaic (PV) panel by an inexpensive polymer collector, thereby reducing the cost of the module and consequently of solar power generation. LSCs have advantages over alternative concentrating systems: they concentrate both direct and diffuse radiation since they are not subjected to a solar concentration ratio limitation [4]; they have a narrow emission band of luminescent species that can be matched to the spectral response of solar cells [5]; they minimize thermalization losses for PV cells [5]; stacking LSC plates containing luminescent species of different absorption characteristics can separate the solar spectrum, and concentrate different parts simultaneously [6]; and they are well suited for building integrated photovoltaic (BIPV) due to their static nature [7-9].

In LSCs, incident solar radiation is absorbed by luminescent material, and subsequently, light is re-emitted over all solid angles. The re- emitted light hitting the waveguide at angles larger than the critical angle is guided via total internal reflection (TIR) to the sheet edges where solar cells are attached. Generally, three surfaces are mirrored to ensure that light can only emerge at the fourth edge where PV cells are located, and an air gap between the LSC sheet and the mirror ensures total internal reflection. LSCs are based on the luminescence down-shifting process therefore there is an inherent loss of energy due to internal energy conversion in the luminescent species, leading to a Stokes-shift in the re-emitted radiation [10]. The overall optical and electrical efficiency of LSCs are limited by re-absorption [10-12], escape cone losses [13] scattering [12], narrow absorption range [14,15], photostability of the luminescent material [16].

Doping concentration of luminescent material and absorption coefficient have a strong influence on determining the efficiency of LSC devices $[12,17]$. The absorption coefficient can be related to the total emission and energy loss processes of re-absorption, scattering and resonance energy transfer (RET) in LSCs. At higher absorption coefficients, quantum yield of the luminescent material is modified through increased non-radiative RET between molecules $[18,19]$, and amplified through re-absorption losses $[11,12]$. In addition; there is a higher

\footnotetext{
* Corresponding author.

E-mail address: schandra@tcd.ie (S. Chandra).
} 


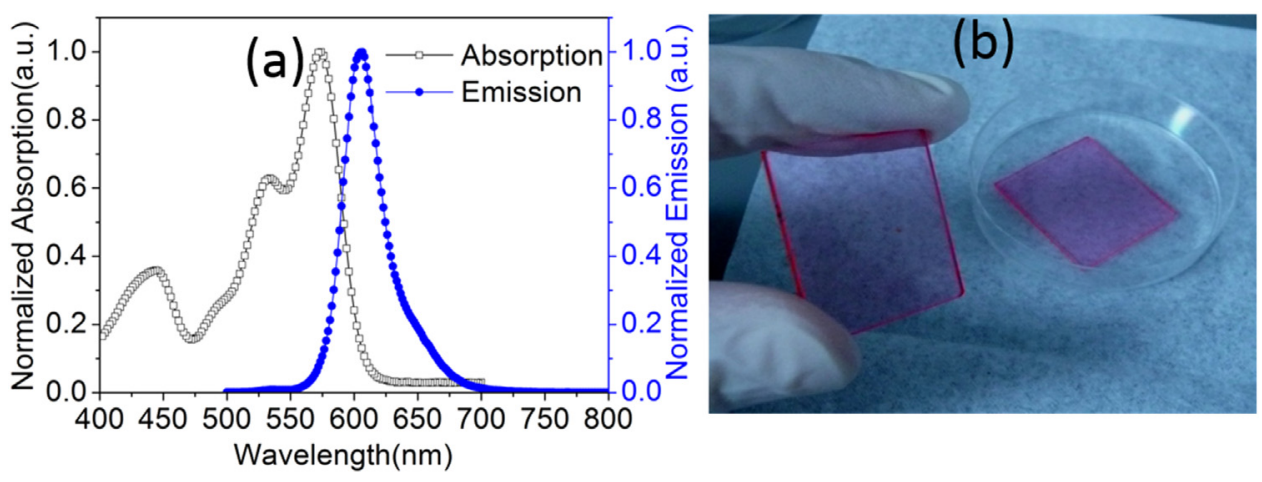

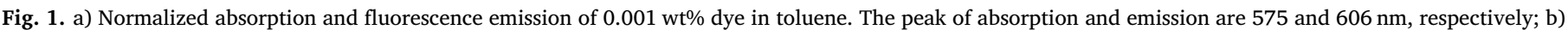
thin film LSC plate fabricated by spin coating of dye doped PMMA polymer on glass substrate.

probability of forming molecule clusters through aggregation of molecules that can induce scattering losses [20]. All these together adversely affect the efficiency of LSC devices. In order to improve the LSCs efficiency, significant advances have been made in terms of the use of quantum dots $[17,21,22]$, rare earth metals [23], aligning luminophore [24], and stacked structured [25], plasmonic interaction improved fluorescence [26], and thin film LSCs [20,27]. The organic dye was replaced with quantum dots in quantum dot solar concentrator [22]. Quantum dots were expected have improved photostability being a semiconductor crystalline structure [28], however, have low fluorescent quantum yield of $20-50 \%$ [29].

LSC systems are divided into two categories based on their thickness; thin film LSCs and bulk LSCs. Decreasing the thickness of LSCs, increase the optical concentration ratio $[15,30]$. Where geometric gain, $\mathrm{G}_{\text {geom }}$ is defined as follows

$\mathrm{G}_{\text {geom }}=\frac{\mathrm{A}_{\text {surface }}}{\mathrm{A}_{\text {edge }}}$

where $\mathrm{A}_{\text {surface }}$ and $\mathrm{A}_{\text {edge }}$ are the areas of the active collector surface and edge, respectively. The geometric gain in combination with optical efficiency $\left(\eta_{\text {opt }}\right)$ determines the theoretical limit of the solar concentration ratio $C_{\text {edge }}$ which is the ratio of irradiance $\left(\mathrm{W} \cdot \mathrm{m}^{-2}\right.$ ) received at the LSC edge to the incident absorbed irradiance;

$\mathrm{C}_{\text {edge }}=\frac{\eta_{\text {opt }} \mathrm{A}_{\text {surface }}}{\mathrm{A}_{\text {edge }}}$

where $\eta_{\text {opt }}$, the optical efficiency, is the ratio of incident radiation delivered to the edge of the LSC. The thin film LSC consists of a thin film of polymer with heavily doped luminescent species (organic dye/ quantum dot/ rare-earth material) coated on highly transparent thicker substrate of the same refractive index to the thin film [15], and fluorescence emission is primarily trapped in the substrate. The bulk LSC has low doping concentration and fluorescence emission trapped in entire volume of LSC.

The luminescent materials quantity in the thin film LSC is intended to be the same as in a comparable size bulk LSC $[31,32]$. Concentrated in a smaller volume of thin film only, hence the thin film LSC has higher absorption coefficient. The refractive index matching between the thin film and the transparent substrate ensures efficient coupling of fluorescence emission from thin film to substrate, where it is trapped and wave-guided by total internal reflection to the edge of the plate, with reduced parasitic losses of re-absorption and scattering [31] compared to bulk LSCs. The reduced scattering and re-absorption losses concept for thin film LSCs has been debated [20], where it has been argued that the gain in optical path-length in the substrate is compensated by the losses within the optically dense thin film.

However, thin film LSCs have several advantages: they enable investigation of stacking LSC plates containing different types of luminescent species [33]; reduced fabrication cost since it requires less doped material [20]; thin films can be coated inside glass and therefore luminescent centres will be protected against UV radiation damage; a very high concentration of luminescent material is easily achieved [8]. This article discusses these arguments through experimentally and predicted results from a ray-trace model.

Monte Carlo ray-trace theoretical model has been applied to optimize bulk LSC device, especially the quantum dot solar concentrator [34-37]. However, this research extends this work and develops a multi-layer ray-tracing model to model thin film LSCs and is validated by comparing predicted and measured results. It provides an accurate tool for quantifying energy loss mechanisms and optimizing thin film LCS for a given set of material parameters. The ray-trace model predicted results are compared with those experimentally obtained.

\section{Material and methods}

Perylene based Lumogen F Red305 dye (BASF, Germany) was used as a luminescent material (referred as dye) - typical absorption and fluorescence emission spectra are presented in Fig. 1a.

Thin film LSCs were fabricated by spin coating (G3 P-8 series spin coater) dye doped acryl-resin polymer (Plexit 55, Carl Roth GmbH + Co. $K G$, Germany) on a clean glass substrate of $25 \times 25 \times 1 \mathrm{~mm}$. The acrylresin polymer Plexit $55\left(5000 \mathrm{mPas}\right.$ at $\left.20^{\circ} \mathrm{C}\right)$ which is a pre-polymerized mixture of $65-70 \%$ MMA, 30-35\% PMMA and $2 \%$ photoinitiator. In the typical process, the highly viscous polymer Plexit 55 was diluted to $25 \mathrm{wt} \%$ in toluene and doped with varying dye doping concentrations from 0.1 to $0.9 \mathrm{wt} \%$. Subsequently the mixture was spin coated on glass substrate to achieve $10 \mu \mathrm{m}$ thin film. Thin films were thermally polymerized for $24 \mathrm{~h}$ at $60^{\circ} \mathrm{C}$ in a vacuum oven to prevent oxygen penetration during polymerization - a fabricated thin film LSC is shown in Fig. 1b. The thickness of the film was $10 \mu \mathrm{m}$ and measured using an atomic force microscope.

\subsection{Optical characterization techniques}

The absorption and fluorescence emission measurements were carried out using a Perkin Elmer Lambda 900 UV/Vis/NIR spectrometer and Perkin Elmer LS55B Luminescence spectrometer, respectively. An AvaSpec-2048 (Avantes, UK) fiber optic spectrometer was used to record the fluorescence emission at the edge of thin film LSCs; the optical setup is illustrated in Fig. 2a. The detector is close to the edge to stop detection of stray fluorescence emission.

A Zeiss LSM 510 confocal laser scanning microscope was used to measure the local fluorescence emission in thin film LSCs. The confocal microscope fluorescence emission is collected from thin film only rather than combined thin film and glass substrate. It is possible to control the depth of the excitation field and eliminate out-of-focus light, because the confocal arrangement allows manipulation of the focal plane. The focal plane was adjusted at the interface of the thin film and glass 


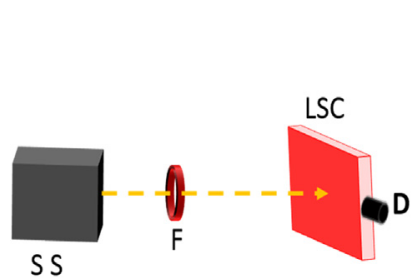

(a)

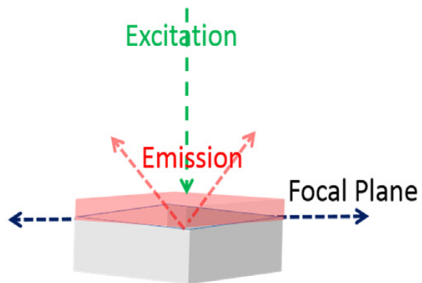

(b)
Fig. 2. The fluorescence emission measurement; a) at the edge of thin film LSCs and the light sources was solar simulator with cut-off filter of $600 \mathrm{~nm}$ (S S; Solar Simulator, F: Filter, D: Detector); b) from top surface of thin film LSCs using a confocal microscope.

substrate by etching thin film thickness; a schematic of the measurement is shown in the Fig. $2 \mathrm{~b}$ where the excitation was by a $488 \mathrm{~nm}$ laser.

The fluorescence lifetime of dye in thin film LSCs was measured using a computer controlled Time Correlated Single Photon Counter (TCSPC) spectrometer FL900 (Edinburgh Instrumentation, UK). The thin film plates were cut to fit a standard sample holder in the instrument (1 cm optical path-length). The nanosecond nF900 flash lamp (FWHM $1 \mathrm{~ns}$ ), was used as an excitation light source with repetition rate of $40 \mathrm{kHz}$, which corresponds to a repetition time of $25 \mu$ s which is higher than the fluorescence life of the dye (ns range)).

\section{Results and discussions}

The optical absorption of thin film LSC plates increased with dye doping concentration in the thin film in Fig. 3a, and their integrated absorbance linearly follows the doping concentration in Fig. 3b. The absorption measurement is based on Beer-lambert law, it is expected to be zero for the blank film, and however, it does have a value which is represented by the intercept. That implies, measured the absorption value is underestimated by artifacts, which could be scattering from dye molecules at higher absorption coefficients.

The fluorescence emission profile at the edge of thin film LSCs, where the solar cell is attached, follows the absorption coefficient up to $162 \mathrm{~cm}^{-1}$. Subsequently, the emission intensity decreased along with the emission peak which is red shifted and the emission shoulder is more pronounced as shown in Fig. 4. The emission peak wavelength is red shifted from $\sim 606 \mathrm{~nm}$ to $\sim 626 \mathrm{~nm}$ as the absorption coefficient is increased from $24 \mathrm{~cm}$ to $202 \mathrm{~cm}^{-1}$ for the thin film LSC.

The locally collected fluorescence emission profile using a confocal microscope is independent of the scan area of the thin film as demonstrated in Fig. 5a \& b which ensures the results are repeatable, reliable, and minimizes the uncertainty in emission measurement for different thin film LSCs.

The local fluorescence emission in the thin film LSCs was measured experimentally and simultaneously modelled using a multilayer raytracing model in Fig. 6a\&b. The fluorescence emission profile is similar to the edge emission apart from there is no red-shift in the emission peak wavelength in Fig. 6.

\subsection{Optimal absorption coefficient of thin film LSCs}

The thin film LSCs solar radiation collection efficiency and its dependence on the doping concentration was examined through electrical characterization. The solar cell was attached to the edge of thin film LSCs, it was uniformly illuminated by a solar simulator at $1 \mathrm{~kW} \mathrm{~m}^{-2}$ and short circuit current $\left(\mathrm{I}_{\mathrm{sc}}\right)$ was measured. The measured $\mathrm{I}_{\mathrm{sc}}$ was compared with spectroscopic, and model predicted results in Fig. 7. Since, detectors have different functionality, hence, the results have been plotted as trends rather than absolute data.

The results established thin film LSCs of $162 \mathrm{~cm}^{-1}$ absorption coefficient had the highest optical and solar radiation collection efficiency. The various results followed each other closely with little deviation at lower absorption coefficients. It appeared that the multilayer ray-trace model was not accurate at absorption coefficients higher than $162 \mathrm{~cm}^{-1}$ in this case. The high absorption coefficient of the thin film implies energy loss processes of scattering, re-absorption, and RET. The combination of these introduce the non-linearity that limited the model for low absorption coefficient thin film LSCs.

\subsection{Analysis of absorption coefficient dependent losses in thin film LSCs}

As Fig. 7 illustrates, thin film LSC efficiency is highly dependent on the absorption coefficient of the luminescent material. The thin film absorption coefficient depends on the volume of luminescent material inside the thin film and follows a linear function of doping concentration for constant optical path-length (thickness of film) as shown in Fig. 3. Ideally, the fluorescence emission should follow this since total emission is function of volume of luminescent material in the thin film
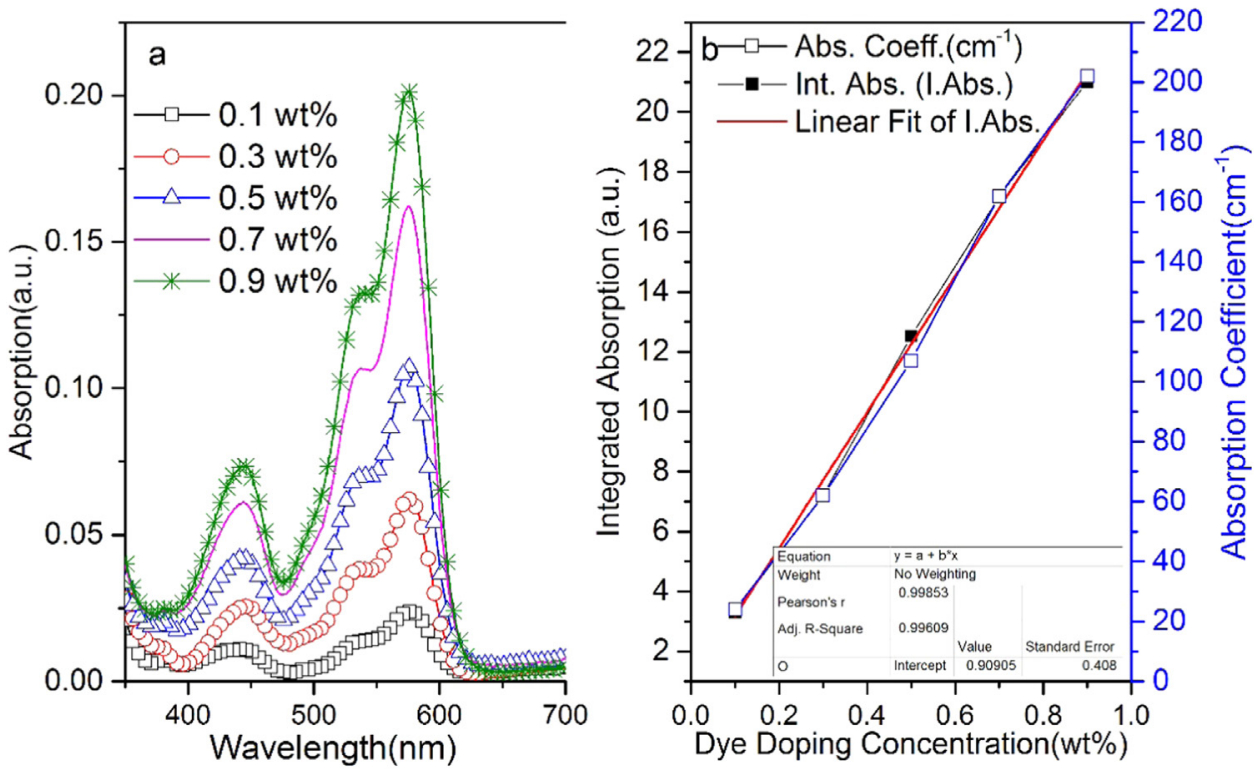

Fig. 3. Optical absorption of thin film LSCs; a) absorption spectra, and b) statistical analysis of integrated absorbance and absorption coefficient. 


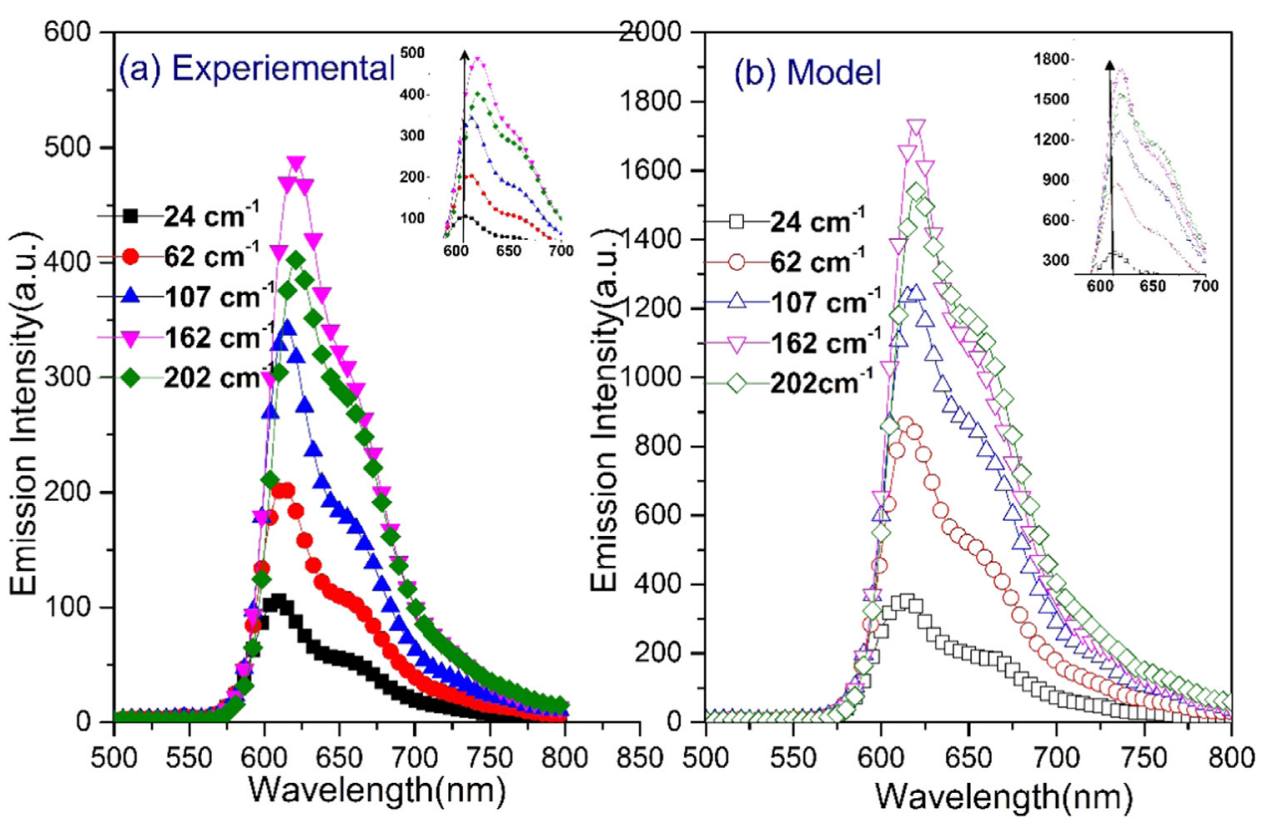

Fig. 4. Fluorescence emission profile at the edge of thin film LSCs plates, a) experimentally measured using fiber optic spectrometer setup, and b) multilayer ray-trace model predicted.

(a)

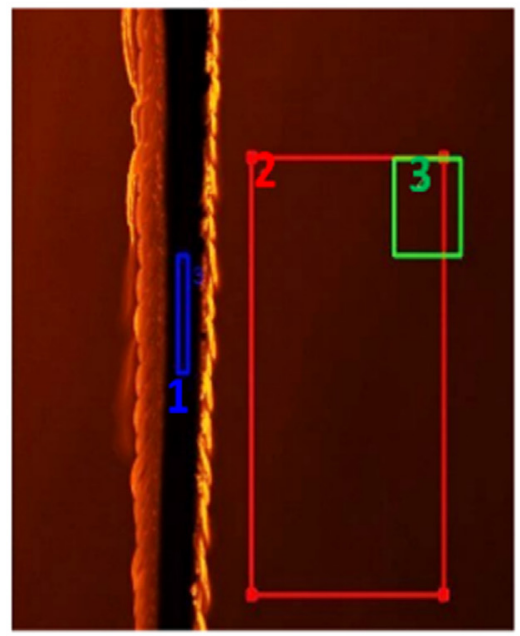

(b)

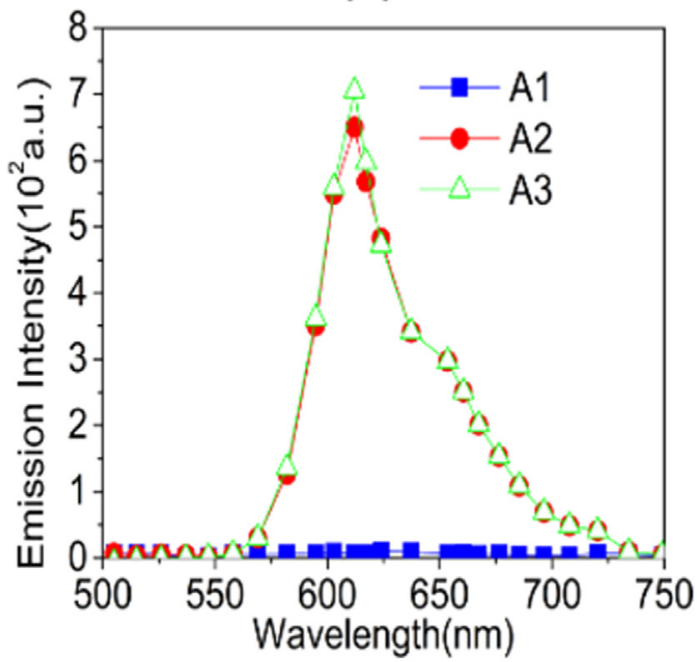

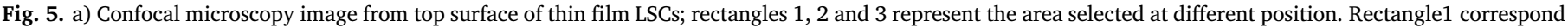
to the background glass substrate, 2 and 3 for dye thin film and their corresponding recorded emission spectra are shown in (b) marked with 1 , 2 , and 3 .

LSCs. However, fluorescence emission of thin film LSC analysis showed deviation from linearity at higher doping concentration as shown in Fig. 8. It is apparent that efficiency is not an infinite function of doping concentration, hence, the overall efficiency is limited and is a nonlinear polynomial function with excellent fitting parameters (Adj. RSquare) close to unity.

The deviation from linearity yields constraints on theoretical raytrace modelling and even establishes limitations for employing the raytrace model to predict the behavior and optimization of thin film LSCs for higher doping concentrations. The model predicted results deviated from experimental for higher than $162 \mathrm{~cm}^{-1}$ absorption coefficient, therefore, higher absorption coefficient results are excluded.

The non-linearity in efficiency can be attributed to energy loss processes within LSCs such as re-absorption, scattering and non-resonant energy transfer. To distinguish and quantify these losses are more challenging due to their interdependence. Such as re-absorption, which inherently depends on the Stokes shift of the luminescent material $[38,39]$ that is the fraction of emission that overlaps with the absorption region as is shown in Fig. 1a. Re-absorption also is a function of optical path-length and absorption coefficient of thin film. Thin film LSC optical path-length is constant since size of these thin film LSCs are invariant. Therefore, re-absorption critically depends on absorption coefficient of this film, which is a direct function of dye doping concentration in the thin film. The fluorescence emission measured from a thin film only and at edge of thin film LSCs can be used to distinguish the optical path-length and absorption coefficient dependent re-absorption. The highest emission profile was deconvoluted to extract information of re-absorption effects caused by the absorption coefficient and optical path length in LSC devices. The deconvolution divided the emission spectra and shows various pronounced peaks in the emission spectrum as shown in Fig. 9.

The locally collected fluorescence emission from the thin film, 


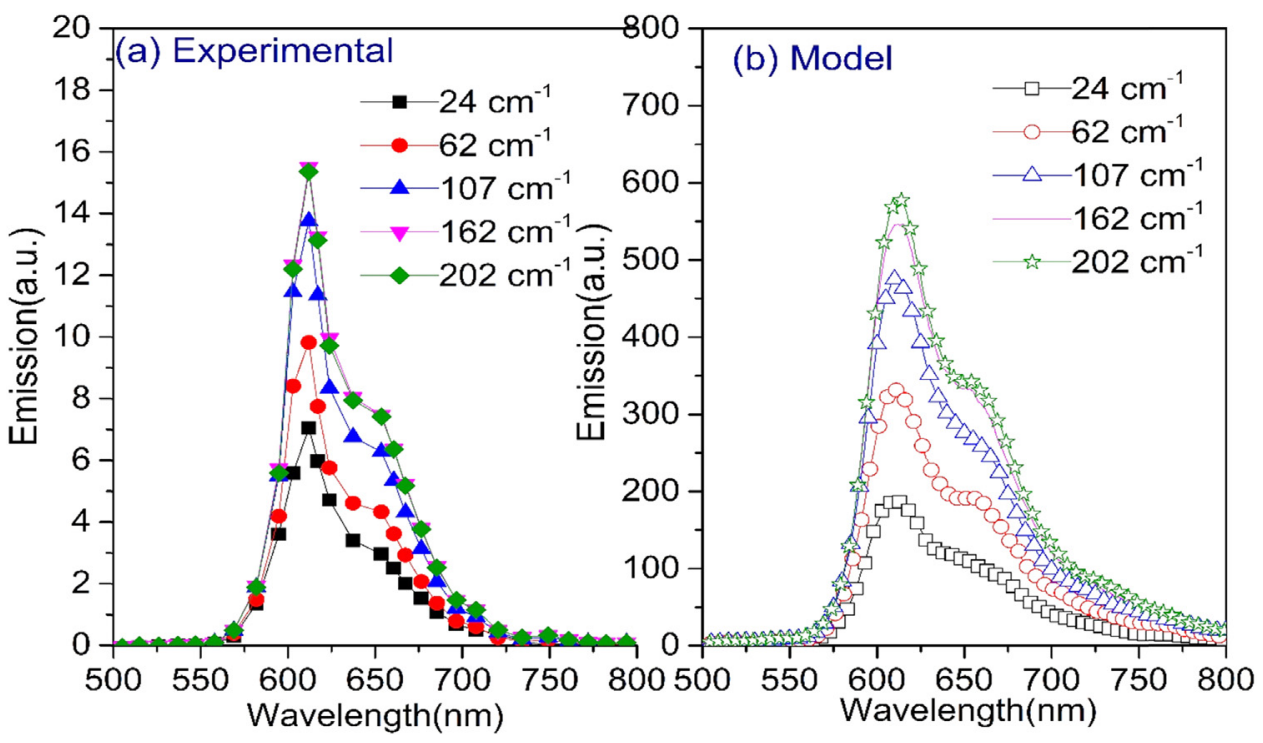

Fig. 6. The local fluorescence emission profile in thin LSCs plates; a) confocal microscope measured, and b) multilayer ray-trace model predicted.

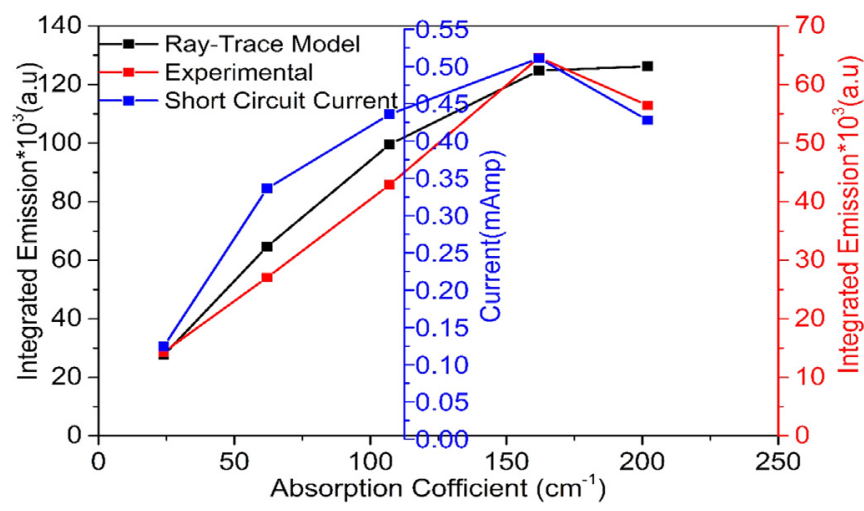

Fig. 7. Fluorescence emission measured and ray-trace model predicted compared with electrical current for thin film LSCs.

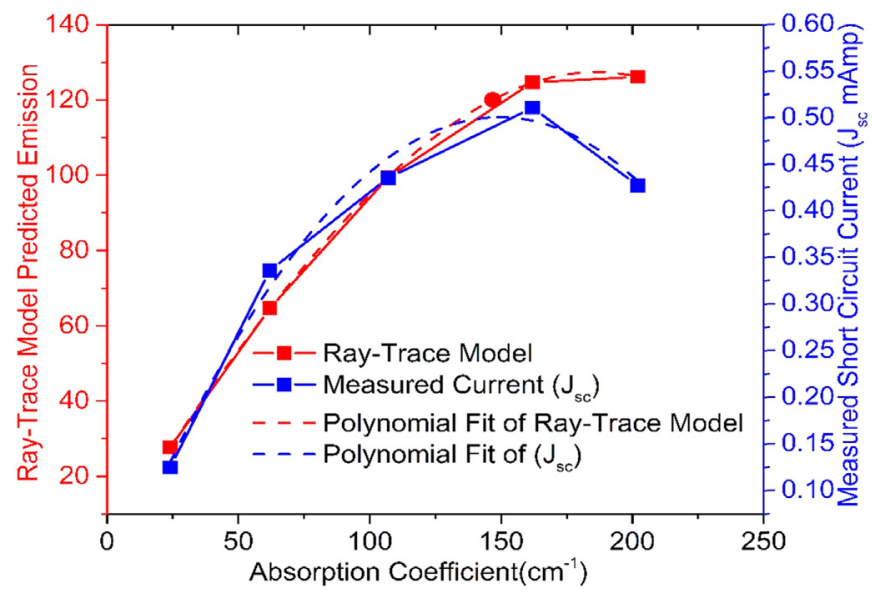

Fig. 8. The total fluorescence emission and optical collection efficiency are non-linear function of red dye concentration in thin film LSCs.

travels through only a very short optical path-length that minimizes the optical path-length depended re-absorption and consequently the redshift. Therefore, their emission profile comprised of two peaks which were similar to emission profile of a very low dye doping solution. At the edge emission profile: fluorescence emission traverses a large optical path-length $(25 \mathrm{~mm})$, and thus has a higher probability of re- absorption, which is more significant as the absorption coefficient is increased. This induced significant red shift in fluorescence emission which is more pronounced with higher absorption coefficient and lead Stokes shift of $20 \mathrm{~nm}$, as presented in the Fig. 4.

In locally collected emission, peak emission wavelength remains same for different absorption coefficient, this indicate there is no reabsorption in Fig. 6. The emission decrease can be attributed to fluorescence RET, supported by a decrease in fluorescence life time as the absorption coefficient of the thin film LSC increased in Fig. 10. At higher absorption coefficients, the spacing between dyes molecules is reduced which increases the possibility of homodimer formation and RET. Since there is a portion of emission overlapping with absorption of dye (Fig. 1a), the formed dimer leads to RET between dye molecules: this type of resonance energy transfer is called energy migration RET $[16,40]$ and consequently there is a decrease in emission for higher absorption coefficients.

\subsection{Thin film LSC and bulk LSCs comparison}

The ray-tracing modelling was employed to model bulk LSCs of the same size as the thin film LSCs. The amount of red dye doping remained constant and was distributed in the bulk LSCs. Since the amount of dye was distributed over a larger volume hence, the absorption coefficient decreased in LSCs compared to thin film LSCs as shown in Fig. 11. The bulk LSCs absorption coefficient is 100 times that of thin film LSCs.

Ray-trace model predicted emission spectra of bulk and thin film LSCs are compared in Fig. 12. The fluorescence emission spectrum and their intensity trend in bulk LSCs deviated from thin film LSCs for absorption coefficient higher than $0.62 \mathrm{~cm}^{-1}$. The increased emission intensity was not proportional to absorption coefficient besides primary emission peak is red shifted and overlap to re-absorption emission peak at absorption coefficient $2.02 \mathrm{~cm}^{-1}$. This is most likely due to a higher re-absorption probability for longer optical path lengths in bulk LSCs. This has led to higher energy losses in the primary emission photon and shifting them to higher wavelength.

The ray-trace model predicted optical parameters for thin film and bulk LSCs are compared in Table 1. It is clear that bulk LSCs have better performance than thin film LSCs in all aspects. Thin film LSCs obtained maximum optical efficiency at $162 \mathrm{~cm}^{-1}$ absorption coefficient, whereas for bulk LSCs dye doping concentration is not high enough to determine the optimum absorption coefficient. Hence, the maximum optical efficiency and concentration ratio is decided by absorption coefficient which is again a function of doping concentration. 

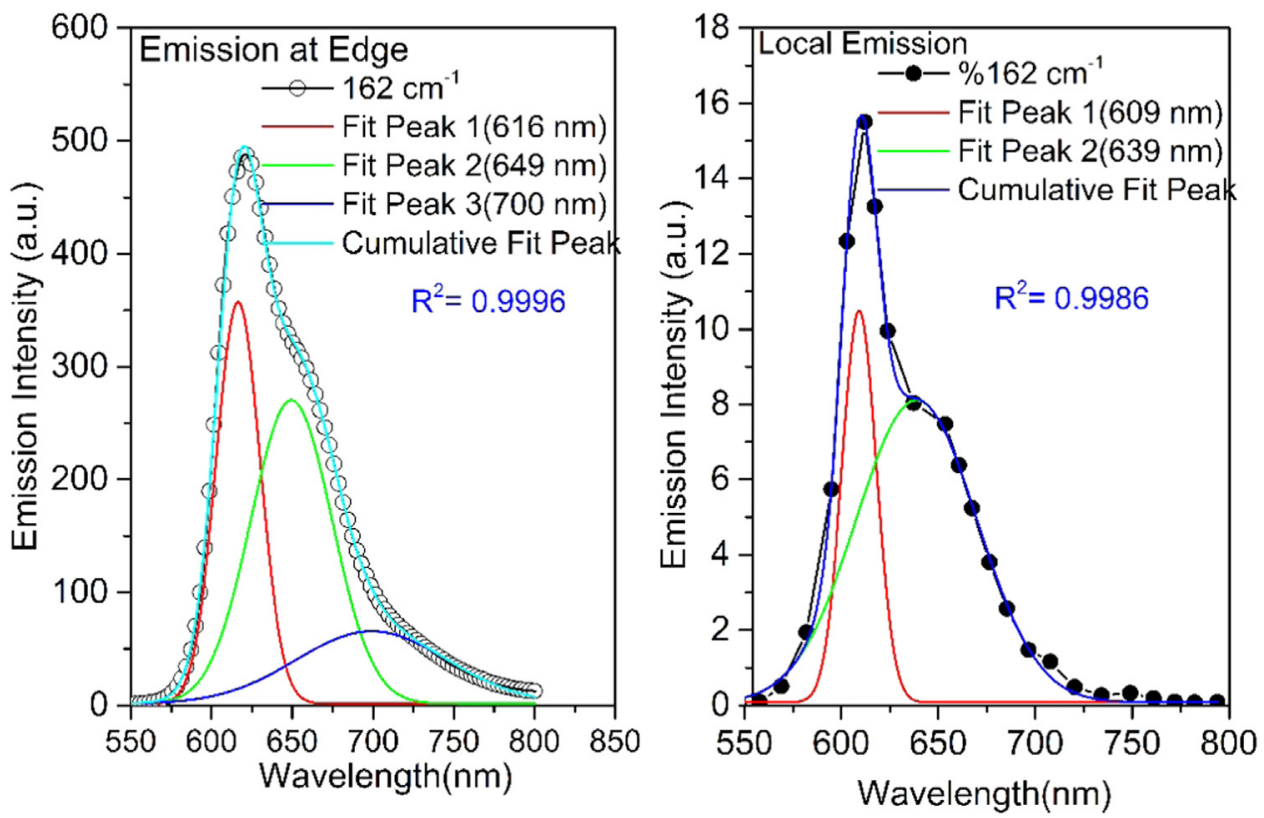

Fig. 9. The deconvolution of emission spectra at the edge and top surface of thin film LSCs. The edge emission contained three distinct peak, and the local top surface emission had two peaks.

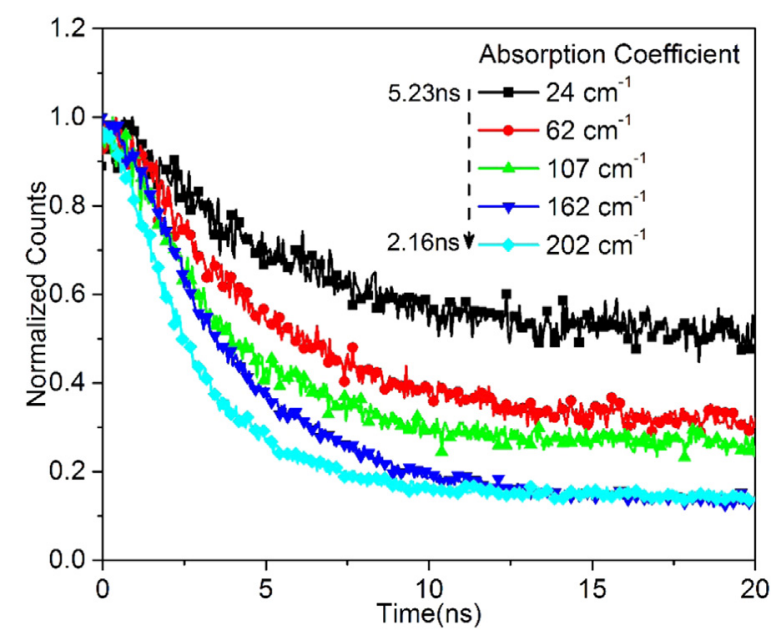

Fig. 10. Fluorescence life time decay rate of red dye in thin film LSCs. (5.23-2.16 ns).

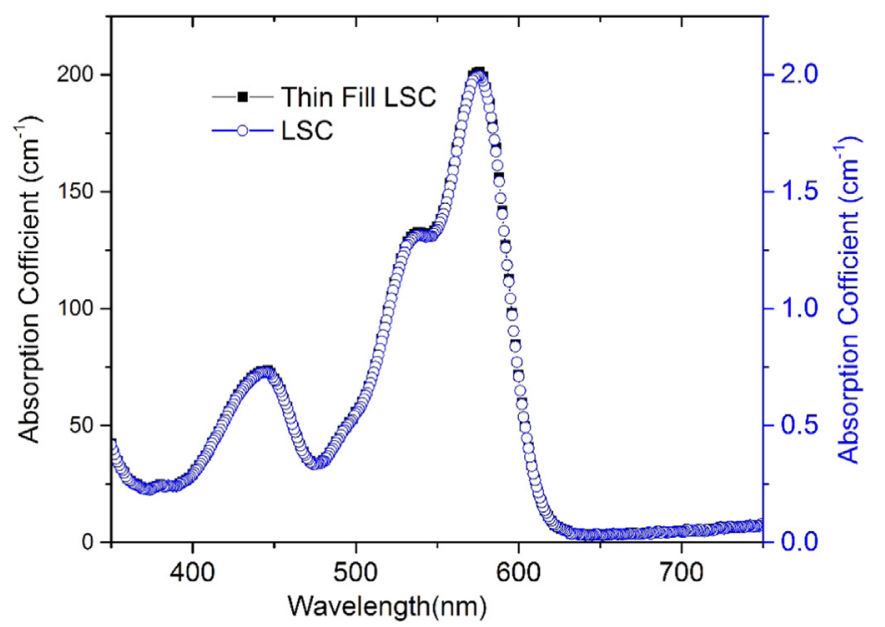

Fig. 11. The absorption coefficient comparison of bulk LSCs and thin film LSCs.

\section{Conclusions}

The spin coating technique was employed to fabricate thin film LSCs of absorption coefficients between 24 and $202 \mathrm{~cm}^{-1}$ by varying red dye doping concentration from 0.1 to $0.9 \mathrm{wt} \%$. The research methodology was established to find the optimum absorption coefficient of thin film LSCs through correlating measured and ray-trace model predicted results. The absorption of the thin film linearly followed the doping concentrations, however, there was a non- zero value at the intercept due to dye molecules causing scattering at higher absorption coefficients. The optical and electrical efficiencies are highly dependent on the absorption coefficient of thin film LSCs device. The efficiencies follow linearly for lower doping absorption coefficients $24-100 \mathrm{~cm}^{-1}$ with the behavior gradually changing to non-linear at higher absorption coefficients. Overall, it is a second order polynomial with inflection point at the optimal absorption coefficient of $162 \mathrm{~cm}^{-1}$. The non-linearity is contributed by energy loss processes of re-absorption, scattering, and RET in thin film LSCs. The multilayer ray-tracing accurately modelled linear LSCs and deviated from experimental results at higher absorption coefficients. The re-absorption and RET energy loss processes are a complex function of absorption coefficient which is separated through locally (thin film) collected and optical path-length fluorescence emission in thin film LSCs. The locally collected fluorescence emission followed linearly at lower values absorption coefficient and overall its non-linear function absorption coefficient, and similarly the optical-path length depend emission profile. However, the emission peak wavelength remained constant in the former, and red-shifted $20 \mathrm{~nm}$ in optical path-length dependent re-absorption. The functionality of locally collected fluorescence emission added and aggregated by RET was confirmed by a decrease in fluorescence life time of dye molecules from 5 to 2 ns for absorption coefficients between 24 and $202 \mathrm{~cm}^{-1}$. In thin film LSCs, re-absorption losses are reduced since the fluorescence emission is confined and wave guided in the substrate of composite through total internal reflection. However, it cannot be completely eliminated and the RET phenomenon is more pronounced and that counters the optical gain achieved through confining and wave guiding the emission in the substrate. This led to non-linearity in thin film LSCs which restricted the performance. It was demonstrated that a similar configuration in a bulk LSC performed better than thin film LSCs. 

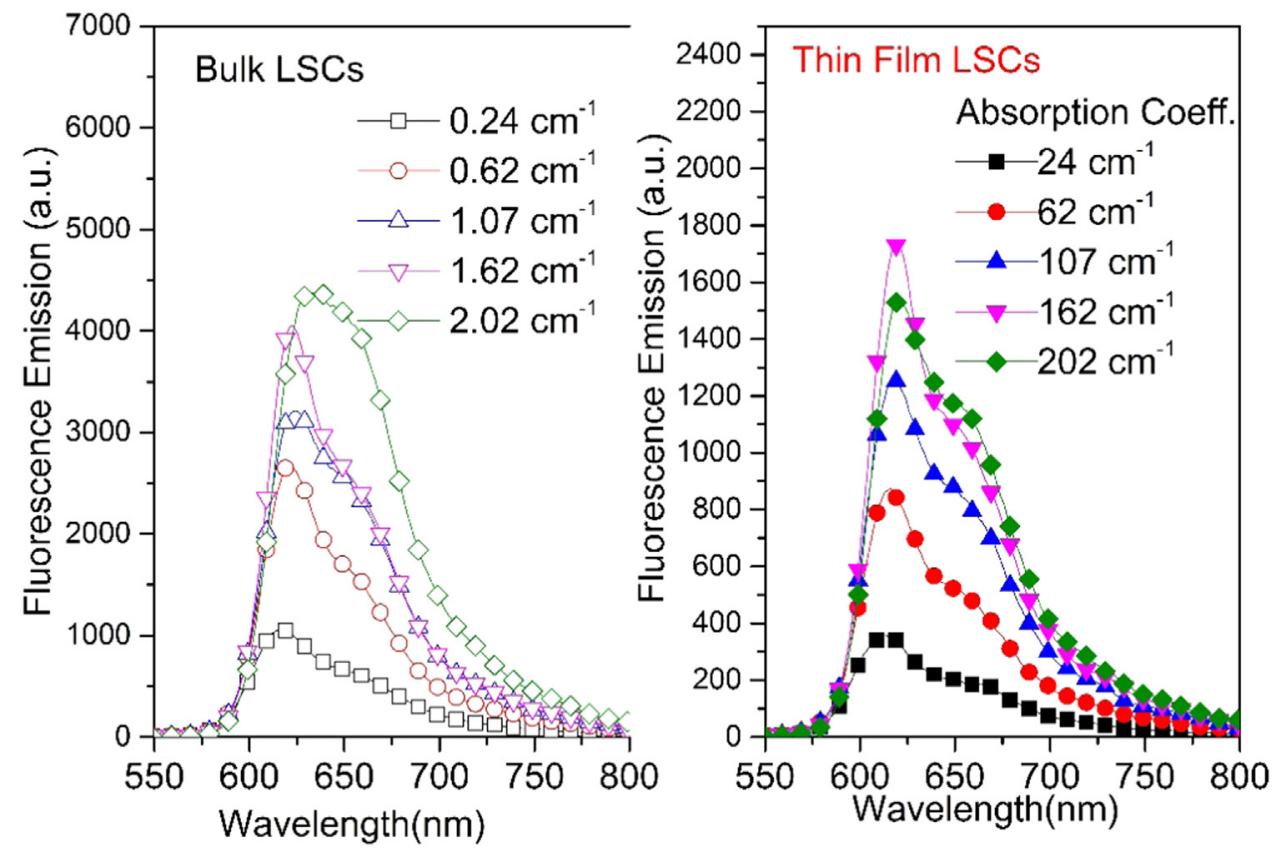

Fig. 12. Ray-trace model predicted fluorescence emission profile at the edge, a) bulk LSCs, and b) thin film LSCs.

Table 1

The optical parameters obtained for LSCs (Opt. C. = Optical Concentration ratio, Opt. Eff. = Optical efficiency of LSCs).

\begin{tabular}{|c|c|c|c|c|c|}
\hline \multicolumn{3}{|l|}{ Thin Film LSCs } & \multicolumn{3}{|l|}{ Bulk LSCs } \\
\hline $\begin{array}{l}\text { Abs. Coefficient } \\
\left(\mathrm{cm}^{-1}\right)\end{array}$ & Opt. C. & Opt. Effi. & $\begin{array}{l}\text { Abs. Coefficient } \\
\left(\mathrm{cm}^{-1}\right)\end{array}$ & Opt. C. & Opt. Effi. \\
\hline 24 & 0.28 & $1.0 \%$ & 0.24 & 1.02 & $3.32 \%$ \\
\hline 62 & 0.66 & $2.66 \%$ & 0.62 & 2.34 & $7.59 \%$ \\
\hline 107 & 1.01 & $4.08 \%$ & 1.07 & 3.22 & $10.34 \%$ \\
\hline 162 & 2.52 & $10.22 \%$ & 1.62 & 3.50 & $11.28 \%$ \\
\hline 202 & 1.26 & $5.14 \%$ & 2.02 & 4.72 & $15.01 \%$ \\
\hline
\end{tabular}

\section{Acknowledgements}

This research work is funded by Higher Education Authority (HEA), Ireland, under the Postgraduate R \& D skills programme and supported by European Research Council under the project Plasmonic Enhancement and Directionality of Emission for Advanced Luminescent Solar Devices (PEDAL) (Project Number 639760)and Science Foundation Ireland

\section{References}

[1] W.H. Weber, J. Lambe, Luminescent greenhouse collector for solar radiation, Appl. Opt. 15 (1976) 3-4.

[2] A. Goetzberger, W. Greube, Solar energy conversion with fluorescent collectors, Appl. Phys. 14 (1977) 123-139.

[3] B.N. Rapp CF, Luminescent Solar Concentrator, in: Proceedings of 13th IEEE PVSC, 1978, pp. 690-693.

[4] G. Smestad, H. Ries, R. Winston, E. Yablonovitch, The thermodynamic limits of light concentrators, Sol. Energy Mater. 21 (1990) 99-111.

[5] J.S. Batchelder, A.H. Zewai, T. Cole, Luminescent solar concentrators 1: theory of operation and techniques for performance evaluation, Appl. Opt. 18 (1979) 3090-3110.

[6] A.A. Earp, G.B. Smith, J. Franklin, P. Swift, Optimisation of a three-colour luminescent solar concentrator daylighting system, Sol. Energy Mater. Sol. Cells 84 (2004) 411-426.

[7] M.G. Debije, P.P.C. Verbunt, Thirty years of luminescent solar concentrator research: solar energy for the built environment, Adv. Energy Mater. 2 (2012) 12-35.

[8] J. Wiegman, E. van der Kolk, Building integrated thin film luminescent solar concentrators: detailed efficiency characterization and light transport modelling, Sol. Energy Mater. Sol. Cells 103 (2012) 41-47.

[9] B. Norton, P.C. Eames, T.K. Mallick, M.J. Huang, S.J. Mccormack, J.D. Mondol,
Y.G. Yohanis, Enhancing the performance of building integrated photovoltaics, Sol, Energy 85 (2011) 1629-1664.

[10] R.W. Olson, R.F. Loring, M.D. Fayer, Luminescent solar concentrators and the reabsorption problem, Appl. Opt. 20 (1981) 2934-2940.

[11] L.R. Wilson, B.C. Rowan, N. Robertson, O. Moudam, A.C. Jones, B.S. Richards, Characterization and reduction of reabsorption losses in luminescent solar concentrators, Appl. Opt. 49 (2010) 1651-1661.

[12] S. Chandra, S.J. McCormack, M. Kennedy, J. Doran, Quantum dot solar concentrator: optical transportation and doping concentration optimization, Sol. Energy 115 (2015) 552-561.

[13] M.G. Debije, P.P.C. Verbunt, B.C. Rowan, B.S. Richards, T.L. Hoeks, Measured surface loss from luminescent solar concentrator waveguides, Appl. Opt. 47 (2008) 6763-6768.

[14] S.W. Leow, C. Corrado, M. Osborn, M. Isaacson, G. Alers, S.A. Carter, Analyzing luminescent solar concentrators with front-facing photovoltaic cells using weighted Monte Carlo ray tracing, J. Appl. Phys. 113 (2013) 214510-243502.

[15] R. Reisfeld, D. Shamrakov, C. Jorgensen, Photostable solar concentrators based on fluorescent glass films, Sol. Energy Mater. Sol. Cells 33 (1994) 417-427.

[16] G. Griffini, L. Brambilla, M. Levi, M. Del Zoppo, S. Turri, Photo-degradation of a perylene-based organic luminescent solar concentrator: molecular aspects and device implications, Sol. Energy Mater. Sol. Cells 111 (2013) 41-48.

[17] S.J. Gallagher, B.C. Rowan, J. Doran, B. Norton, Quantum dot solar concentrator: device optimisation using spectroscopic techniques, Sol. Energy 81 (2007) $540-547$.

[18] A.F. Mansour, H.M.A. Killa, S. Abd El-Wanees, M.Y. El-Sayed, Laser dyes doped with poly(ST-Co-MMA) as fluorescent solar collectors and their field performance, Polym. Test. 24 (2005) 519-525.

[19] L.R. Wilson, B.S. Richards, Measurement method for photoluminescent quantum yields of fluorescent organic dyes in polymethyl methacrylate for luminescent solar concentrators, Appl. Opt. 48 (2009) 212-220.

[20] G. Griffini, M. Levi, S. Turri, Thin-film luminescent solar concentrators: a device study towards rational design, Renew. Energy 78 (2015) 288-294.

[21] M. Kennedy, S.J. Mccormack, J. Doran, B. Norton, Improving the optical efficiency and concentration of a single-plate quantum dot solar concentrator using near infrared emitting quantum dots, Sol. Energy 83 (2009) 978-981.

[22] K. Barnham, J. Marques, J. Hassard, P. O'brien, Quantum-dot concentrator and thermodynamic model for the global redshift, Appl. Phys. Lett. 76 (2000) $1197-123114$.

[23] S.F.H. Correia, V. Onica De, Z. Bermudez, S.J.L. Ribeiro, P.S. And, R.A.S. Ferreira, L. Is, D. Carlos, Luminescent solar concentrators: challenges for lanthanide-based organic-inorganic hybrid materials, Mater. Chem. A 2 (2014) 5580-5596.

[24] R. Rowan, W. Mac Queen, Yuen Yap Cheng, A. el G.C.R. Clady, T. Schmidt, Towards an aligned luminophore solar concentrator, Liq. Cryst. Adv. Funct. Mater. Chem. Ber. 19 (2009) 1-6.

[25] J.C. Goldschmidt, M. Peters, A. Bö, H. Helmers, F. Dimroth, S.W. Glunz, G. Willeke, Increasing the efficiency of fluorescent concentrator systems, Sol. Energy Mater. Sol. Cells 93 (2008) 176-182.

[26] S. Chandra, J. Doran, S.J. McCormack, M. Kennedy, A.J. Chatten, Enhanced quantum dot emission for luminescent solar concentrators using plasmonic interaction, Sol. Energy Mater. Sol. Cells 98 (2012) 385-390.

[27] S. El-Bashir, F. Barakat, M. AlSalhi, Double layered plasmonic thin-film luminescent solar concentrators based on polycarbonate supports, Renew. Energy 63 (2014) 
642-649.

[28] A.P. Alivisatos, Perspectives on the physical chemistry of semiconductor nanocrystals, J. Phys. Chem. 100 (1995) 13226-13239.

[29] B.O. Dabbousi, J. Rodriguez-Viejo, F.V. Mikulec, J.R. Heine, H. Mattoussi, R. Ober, K.F. Jensen, M.G. Bawendi, (CdSe)ZnS core - shell quantum dots: synthesis and characterization of a size series of highly luminescent nanocrystallites, J. Phys. Chem. B 101 (1997) 9463-9475.

[30] G. Griffini, M. Levi, S. Turri, Novel crosslinked host matrices based on fluorinated polymers for long-term durability in thin-film luminescent solar concentrators, Sol. Energy Mater. Sol. Cells 118 (2013) 36-42.

[31] T. Dienel, C. Bauer, I. Dolamic, D. Brü, Spectral-based analysis of thin film luminescent solar concentrators, Sol. Energy 84 (2010) 1366-1369.

[32] G. Griffini, M. Levi, S. Turri, Novel high-durability luminescent solar concentrators based on fluoropolymer coatings, Prog. Org. Coat. 77 (2014) 528-536.

[33] M. Carlotti, G. Ruggeri, F. Bellina, A. Pucci, Enhancing optical efficiency of thin-film luminescent solar concentrators by combining energy transfer and stacked design, J. Lumin. 171 (2016) 215-220.

[34] M. Kennedy, Monte-Carlo Ray-Trace Modelling of Quantum Dot Solar
Concentrators, Dublin Institute of Technology, Dublin, Ireland, 2009.

[35] A. Kerrouche, D. Hardy, D. Ross, B. Richards, Luminescent solar concentrators: from experimental validation of 3D ray-tracing simulations to coloured stained-glass windows for BIPV, Sol. Energy Mater. Sol. Cells 122 (2014) 99-106.

[36] S.J. Gallagher, P.C. Eames, B. Norton, Quantum dot solar concentrator behaviour, predicted using a ray trace approach, Int. J. Ambient Energy 25 (2004) 47-56.

[37] A.R. Burgers, L.H. Slooff, R. Kinderman, J.A.M. van Roosmalen, Modelling of luminescent concentrators by ray-tracing, in: Proceedings the 20th European Photovoltaic Solar Energy Conference Exhibition, 2005, pp. 394-397.

[38] J.R. Lakowicz, Principles of Fluorescence Spectroscopy, 3rd edition, Springer, USA, New York, 2006.

[39] A. Bozzola, V. Robbiano, K. Sparnacci, G. Aprile, L. Boarino, A. Proto, R. Fusco, M. Laus, L.C. Andreani, D. Comoretto, A multi-optical collector of sunlight employing luminescent materials and photonic nanostructures, Adv. Opt. Mater. 4 (2016) 147-155.

[40] M.N. Berberan-Santos, B. Valeur, Fluorescence depolarization by electronic energy transfer in donor-acceptor pairs of like and unlike chromophores, J. Chem. Phys. 95 (1991) 8048. 